\title{
Quasilineare elliptische Differentialgleichungen zweiter Ordnung in mehrdimensionalen Gebieten mit.Kegelspitzen
}

\author{
E. Mrersemann
}

Wir untersuchen Regularitätseigenschaften der Lösungen $u$ einer Klasse quasilinearır elliptischer Randwertáufgaben zweiter Ordnung in $n$-dimensionalen Gebieten mit Kegelspitzen. Wir beweisen : $u \in C^{1, \lambda} \cap H^{2}$ in einer Umgebung der Spitze.

Исследуется вопрос о регулярности решения и для некоторых квазилинейых эллиптически краевых задач для многомериых областей с коническими точками. Доказывается, что $u \in C^{1, \lambda} \cap H^{2}$ в окрестности вершины.

We study regularity properties for solutions $u$ of a class of quasilinear elliptic boundary value problems of second order in $n$-dimensional domains with conic vertices. We prove $u \in C^{1, \lambda} \cap H^{2}$ near the vertex.

\section{Einleitung und Resultate}

Jas Regularitätsverhalten der Lösungen einer Klasse quasilinearer elliptischer Gleichungen zweiter Ordnung in den Ecken zweidimensionaler Gebiete mit stückweise glattem Rand wurde in [10] untersucht. Mit Hilfe der Barrierenmethode konnte unter einfachen Annahmen gezeigt werden, daß die ersten Ableitungen der Lösung auch in den Ecken einer Hölder-Bedingıng genügen, wenn für die entsprechenden Innenwinkel $\gamma$ gilt $0<\gamma<\pi$. In dieser Mitteilung behandeln wir mehrdimensionale Aufgaben. Wie schon in $[10]$ benutzen wir eine elementare Barrierefunktion. Azzar $[1,2]$, AzzAM und KREYSzIG [3] und DzIUK [4] studierten damit lineare und semilineare Aufgaben.

Es sei $\Omega \subset \mathbf{R}^{N}$ ein beschränktes und einfach zusammnenhängendes Gebiet. Wir betrachten das Randwertproblem

$$
\begin{aligned}
\int_{\Omega} \sum_{i=1}^{N} a_{i}\left(u_{x}\right) \varphi_{x_{i}} d x & =\int_{\Omega} f \varphi^{\prime} \cdot d x \text { für alle } \varphi \in C_{0}{ }^{\infty}(\Omega), \\
u & =\Phi \text { auf } \partial \Omega .
\end{aligned}
$$

Dabei sind $f \in H^{-1}(\Omega), \Phi \in H^{1}(\Omega)$ gegebene Funktionen, $a(p)=\left(a_{1}(p), \ldots, a_{N}(p)\right)$, $p=\left(p_{1}, \ldots, p_{N}\right)$, ist ein stark monotones und koerzitives $C^{1}$-Vektorfeld.

Das Vektorfeld $a(p)$ heilit sturk monoton und koerzitiv von der Klasse $C^{\mathbf{1}}$, wenn gilt:

$$
\begin{aligned}
& a_{i}(p) \in C^{\mathbf{D}}\left(\mathbf{K}^{N}\right) \\
& \left(a_{i}(p)-a_{i}(q)\right)\left(p_{i}-q_{i}\right) \geqq v|p-q|^{2} \quad \text { für alle }
\end{aligned}
$$


$p, q \in \mathbf{R}^{N}$ mit einer von $p, q$ unabhängigen positiven Konstanten $\nu$. Es ist $|p|=\left(p_{1}{ }^{2}+\right.$ $\left.\cdots+p_{N}^{2}\right)^{1 / 2}$.

$$
\begin{aligned}
& \left|a_{i j}(p)\right| \leqq M \text { für alle } p \in \mathbf{R}^{N}(M<\infty \text { und unabhängig von } p) \text {, wobei } \\
& a_{i j}=\frac{\partial a_{i}}{\partial p_{j}} \text { gesetzt ist. }
\end{aligned}
$$

Für diese Definition vergleiche man etwa [7: Chapter 3.4]. Aus (2)-(4) folgt (s. z. B. [7: S. 100]):

$$
a_{i j}(p) \xi_{i} \xi_{j} \geqq \nu|\xi|^{2} \text { für alle }{ }^{-} p, \xi \in \mathbf{R}^{N}
$$

Über doppelt auftretende Indizes wird sumniert.

Die bekannten Sobolewschen Banachräume, die als Vervollständigung von $C^{m}(\Omega)$ in der ühlichen Sobolew-Norm definiert werden, bezeichnen wir mit $H_{s}{ }^{m}(\Omega)(s>1$; $m=1,2$ ). Wir setzen $H^{m}=H_{2}{ }^{m}$.

Dic Aufgabe (1) hat unter den obigen Ànnahmen eine eindeutig bestimmte I,ösung $u \in H^{1}(\Omega)$ (s. z. B. [6]).

Das Verhalten der Lösungen quasilinearer elliptischer Gleichungen zweiter Ordnung am Rand von zwei -und dreidimensionalen nichtglatten Gebieten wurde-auch von Tolksdorf [13, 14] untersucht. Für zweidimensionale Gebiete studiért Tolksdorf den Fall, daß die Innenwinkel in den Ecken größer als $\pi$ sind. Für eine Klasse von Aufgaben konnte gezeigt werden, daß die Lösungen in den Ecken einer Hölder-Bedingung genügen. Außerdem werden in $[13,14]$ Abschätzungen fïr den Hölder-Exponenten angegeben. Für lineare elliptische Differentialgleichungen in Gebieten mit nichtglattem Rand findet man bei GRISvarD [5] zahlreiche Literaturhinweise.

Das Gebiet $\Omega$ habe im Ursprung des $x$-Koordinatensystems eine Spitze. Der Rand sei außer in 0 glatt in einer Umgebung des Ursprungs. Es wird gesetzt:

$$
\Omega_{\varrho}=\Omega \cap B_{\varrho}(0), \quad 0<\varrho \leqq \varrho_{1}, \quad \varrho_{1}>0 \text { genügend klein. }
$$

Dabei ist $B_{e}(0)=\{x|| x \mid<\varrho\}$. Wir nehmen gleich an, daß $\partial \Omega \cap B_{\rho}(0)$ in einem Kegelmantel des folgenden Typs enthalten ist:

$$
\begin{aligned}
& x_{N}=h\left(x^{\prime}\right), \quad x^{\prime}=\left(x_{1}, \ldots, x_{N-1}\right), \quad \text { mit } \\
& h \in C^{2}\left(\mathbf{R}^{N-1} \backslash\{0\}\right), \quad h\left(t x^{\prime}\right)=t h\left(x^{\prime}\right) \quad \text { für alle } t \geqq 0 \\
& \text { und } h\left(x^{\prime}\right) \geqq \alpha\left|x^{\prime}\right|, \quad \alpha>0 .
\end{aligned}
$$

Dann ist

$$
\Omega_{e}=\left\{x\left|x_{N}>h\left(x^{\prime}\right), \quad\right| x \mid<-\varrho\right\} .
$$

Im allgemeinen Fall muß man das Eckgebiet mit einem Tangentialkegel der Form (6) auf diesen abbilden. Man vergleiche dazu bei Kondrat'Jev [9].

.Es sei $\left|D^{2} \Phi\right|=\sum_{i, j=1}^{N}\left|\Phi_{x_{i} x_{j}}\right|$. Unter den Voraussetzungen $(2)-(4),(6)$ und

$$
\begin{aligned}
& \Phi \in H_{\delta}^{2}\left(\Omega_{e}\right) \text { mit } s>N, \\
& |f| \leqq c|x|^{x-1} \text { : und }\left|D^{2} \Phi\right| \leqq c|x|^{x-1} \quad(x>0) \text { für } x \in \Omega_{e},
\end{aligned}
$$

hat man das folgende Resultat.

Theorem: Für die Lösung u von (1) gilt $u \in H^{2}\left(\Omega_{e / 2}\right) \cap C^{1, \lambda}\left(\bar{\Omega}_{e / 2}\right), 0<\lambda<1$.

Bemerkungen: 1. Das Theorem bleibt richtig, wenn im dreidimensionalen Fall der Kegel Kanten $S_{l}$ enthält, deren Innenwinkel $\gamma_{1}$ der Bedingung $0<\gamma_{l}<\pi$ ge- 
nügen. Darüber hinaus müssen Ungleichungen des 'Lyps (8) längs der Kanten angenommen werden. Man vergleiche hierzu für den linearen Fall AzzaM [2].

2. Mit Hilfe des obigen Satzes läßt sich die Existenz von Lösungen $u \in C^{1, \lambda}(\bar{\Omega})$ des mehrdimensionalen nichtparametrischen Minimalflächenproblems in gewissen (zum Beispiel konvexen) Gebieten mit Ecken und Kanten beweisen. Man vergleiche dazu $[10,11]$ für den zweidimensionalen Fall.

\section{Beweis des Theorems}

Wir führen Kugelkoordinaten ein: $x_{k}=r \xi_{k} \sin \theta$ fiir $1 \leqq k \leqq N-1$ und $x_{N}=r$ $\times \cos 0, N \geqq 3$. Dabei ist

$$
\begin{aligned}
\xi_{1} & =\sin \varphi_{N-2} \ldots \sin \varphi_{2} \sin \varphi_{1}, \\
\xi_{2} & =\sin \varphi_{N-2} \ldots \sin \varphi_{2} \cos \varphi_{1}, \\
\xi_{3} & =\sin \varphi_{N-2} \ldots \sin \varphi_{3} \cos \varphi_{2}, \\
& \vdots \\
\xi_{N^{\prime}-2} & =\sin \varphi_{N-2} \cos \varphi_{N^{\prime}-3}, \\
\xi_{N^{\prime}-1} & =\cos \varphi_{N-2},
\end{aligned}
$$

und $0 \leqq 0 \leqq \frac{\pi}{2}, 0 \leqq \varphi_{1} \leqq 2 \pi, 0 \leqq \varphi_{i}<\pi$ für $2 \leqq i \leqq N_{:}-2$ : Wegen (6) gilt für $0_{0}=\max \left\{\theta \mid x \in \Omega_{e}\right\}$ die Ungleichung

$$
0<\theta_{0}<\frac{\pi}{2}
$$

Fiir die Funktion

$$
W=A r^{1+\mu} \cos \lambda \theta+\Phi_{x_{i}}(0) x_{i}+\Phi(0)
$$

mit $A=$ const $>0,0<\mu<1$ und $\lambda>1$ hat man $W>0$ in $\bar{\Omega}_{e} \backslash\{0\}$ aufgrund von (9) und (7), wenn $\lambda-1$ und $\mu$ hinreichend klein sind. Wir setzen

$$
L W=u_{i j}\left(W_{x}\right) W_{x_{i} x_{j}} .
$$

Dabei können wir $a_{i j}=a_{i i}$ annehmen (sonst ersetzt man $a_{i j}$ durch $1 / 2\left(a_{i j}+u_{j i}\right)$ ).

T,emma: Es gibt ein $l>0$, so daß für jedes $\lambda$. mit $0<\lambda-1 \leqq d$ positive Zahlen $\eta(i)$ und $\mu_{0}(\lambda)$ existieren mit $L W \leqq-A \eta r^{\mu-1}$. für alle $0<\mu<\mu_{0}$.

Beweis: Nach einer elementaren Rechnung erhalten wir

$$
W_{x_{1} x_{j}}=A r^{\mu-1} V_{i j}, \quad V_{i j}=V_{j i} .
$$

Es ist

$$
\begin{aligned}
V_{i j}= & \xi_{i} \xi_{j} \cos ^{2} \theta \cos \lambda \theta-(\lambda \cot \theta \sin \lambda .0-\cos \lambda \theta)\left(\delta_{i j}-\xi_{i} \xi_{j}\right)-\lambda^{2} \xi_{i} \xi_{j} \cos ^{2} \theta \cos \lambda .0 \\
& +\dot{R}_{i j} \text { fiir } 1 \leqq i, j \leqq N-1,
\end{aligned}
$$

$V_{i N}=-\xi_{i} \sin \theta \cos \theta \cos \lambda \theta+\lambda^{2} \xi_{i} \sin \theta \cos \theta \cos \lambda .0+R_{i N} \quad$ für $\quad 1 \leqq i \leqq N-1$

und

$$
V_{N i}=\sin ^{2} \theta \cos \lambda \theta-\lambda^{2} \sin ^{2} \theta \cos \lambda \theta+R_{N N}
$$


Für die Reste $R_{i j}$ gilt $R_{i j}=R_{i i}$ und

$$
\left|R_{i j}\right| \leqq \mu \lambda+\frac{1}{2}\left(1+\delta_{i j}\right)\left(2 \mu+\mu^{2}\right), \quad 1 \leqq i, \quad j \leqq N
$$

Wir setzen

$$
\begin{aligned}
& i^{2}=(1+\tau)^{2}, \quad \tau>0 ; \quad \eta_{i}=\xi_{i} \cos \theta \quad \text { für } \quad 1 \leqq i \leqq N-1, \\
& \eta_{N}=-\sin \theta .
\end{aligned}
$$

Dann ist

$$
\begin{gathered}
a_{i j} V_{i j}=-2 \tau \sum_{i, j=1}^{N} a_{i j} \eta_{i} \eta_{j} \cos \lambda \theta-(\lambda \cot \theta \sin \lambda \theta-\cos \lambda \theta) \sum_{i, j=1}^{N-1} a_{i j}\left(\delta_{i j}-\xi_{i} \xi_{j}\right)+Q, \\
|Q| \leqq \frac{1}{2} M N(N+1)\left(\tau^{2}+2 \mu+\mu^{2}\right)+M N^{2} \lambda_{i} \mu .
\end{gathered}
$$

Für $0<\tau \leqq \tau_{0}, \tau_{0}$ hinreichend klein, ist bei $x \in \Omega_{e}$ der Faktor von der zweiten Summe nicht negativ, und man hat wegen (9)

$$
\min _{\substack{0 \leqq \mathrm{r} \\ 0 \leqq 0 \leqq r_{0}}} \cos (1+\tau) 0 \geqq c_{0}>0 \text {. }
$$

Wenn wir $c_{1}=\frac{1}{2} M N(N+1)$ und $c_{2}=\frac{3}{2} M N(N+1)+M N^{2}\left(1+\tau_{0}\right)$ setzen, dann folgt aus $(5)$ die Ungleichung

$$
a_{i j} \dot{V}_{i j} \leqq-2 v c_{0} \tau+c_{1} \tau^{2}+c_{2} \mu
$$

für alle $0<\tau \leqq \tau_{0}$ und für alle $0 \leqq \mu \leqq 1$. Dabei ist zu beachten, da $\$$ aufgrund von (5) gilt

$$
a_{i j}\left(\delta_{i j}-\xi_{i} \xi_{j}\right)=\sum_{i=1}^{N-1} \lambda_{i}-a_{i j} \xi_{i} \xi_{j} \geqq \sum_{i=1}^{N-2} \lambda_{i} \geqq(N-2) \nu .
$$

Die Zahlen $\lambda_{i}$ bezeichnen hier die Eigenwerte der Matrix $\left[a_{i j}\right], 1 \leqq i, j \leqq N-1$. Der größte Eigenwert ist $\lambda_{N-1}$. Wir erhalten schließlich

$$
a_{i j} V_{i j} \leqq-\nu c_{0} \tau+c_{2} \mu
$$

für alle $0 \leqq \mu \leqq 1$ und für alle $0<\tau \leqq \min \left(\tau_{0}, \frac{\nu c_{0}}{c_{1}}\right)$ 】

Bemerkung: K. MrLer [12] studiert allgemeinere Ansätze für W. Für unser Problem kommt man mit dem obigen speziellen Ansatz aus. Dadurch vereinfachen sich die Rechnungen wesentlich und die Abhängigkeit der Konstanten $\eta, \mu$ von $\lambda$ läßt sich genauer charakterisieren.

Das Theorem folgt dann wie im zweidimensionalen Fall [10: Satz 2.4]. Dabei wird wesentlich eine a priori-Abschätzung für quasilineare elliptische Differentialgleichungen zweiter Ordnung für reguläre Gebiete eingesetzt. Mit Hilfe eines Lokalisierungsverfahrens, man vergleiche bei Kondra'T'JEv [8], kann man die Kegelspitze ausschöpfen. Das obige Lemma garantiert dann, daß die Lösung $u$ auch im Abschluß einer Umgebung der Spitze zur Klasse $C^{1, \alpha}$ gehört, $0<\alpha<1$. 


\section{LTTERATUR}

[1] AzZAM, A.: Behaviour of solutions of Dirichlet problem for elliptic equations at a corner. -Indian J. Pure Appl. Math. 10 (1979), 1453-1459.

[2] AzZAM, A.: On Dirichlet's problem for elliptic equations in sectionally smooth $n$-dimensional domains. SIAM J. Math. Anal. 11 (1980), 248-253.

[3] Azzam, A., und E. Kreyszig: Über das gemischte Randwertproblem für elliptische Gleichungen in $n$-dimensionalen Gebieten mit Kanten. Ann. Acad. Sci. Fenn. Ser. AI Math. 5 (1980), $341-346$.

[4] Dzıuk, G.: Das Verhalten von Lösungen semilinearer elliptischer Systeme an Ecken eines Gebietes. Math. Z. 159 (1978), 89-100.

[5] Grisvard, P.: Behaviour of the solutions of an elliptic boundary value problem in a poly. gonal or polyhedral domain. Numerical Sol. of Part. Diff. Equ. III, New York 1976, pp. $207-274$.

[6] Hartias, P., and G. Stanipacchia: On some nonlinear elliptic differential functional equations. Acta Math. 115 (1966), 153-188.

[7] KiNderlehrer, D., and G. Stampaccha: An Introduction to Variational Inequalities and Their Applications. New York 1980.

[8] КондРАтьев, В. А.: ІІраевые задачи пля әллиптических уравнений в областях с коническими или угловыми точками. Труды Моск. матем. о-ва 16 (1967), 208-292.

[9]. Кондрать Ев, В. А.: Краевые задачи для параболических уравнения в замкнутых областях. Труды Моск. матем. о-ва 15 $(1966), 400-451$.

[10] Miersemanis, R.: Zur Regularität verallgemeinerter Lösungen von quasilinearen elliptischen Differentialgleichungen zweiter Ordnung in Gebieten mit Ecken. Z. Anal. Anw. 1 (1982), $59-71$.

[11] Mersentas, E.: Zur Gleichung der Fläche mit gegebener mittlerer Krümmung in zweidimensionalen eckigen Gebieten. Math. Nachr. 110 (1983), 231-241.

[12] Mrleer, K.: Extremal barriers on cones with Phragmén-Lindelöf theorems and other applications. Ann. Mat. Pura Appl. 90 (1971), $297-329$.

[13] Tolksdorf, P.: On quasilinear boundary value problems in domains with corners. Nonlinear Anal. 5 (1981), $721-735$.

[14] Tolksdorf, P.: On the behaviour near the boundary of solutions of quasilinear equations. Analysis (to appear).

Manuskripteingang: 18.11.1981

\section{VERFASSER:}

Doz. Dr. Erich Miersemiani

Sektion Mathematik der Karl-Marx-Universität

DDR - 7010 Leipzig, Karl-Marx-Platz 